\title{
Linking Theory of Mind in Human-Agent Interactions to Validated Evaluations
}

\author{
Can Explicit Questionnaires Measure Implicit Behaviour?
}

\author{
Evelien Heyselaar ${ }^{\dagger}$ \\ Communication \& Media \\ Behavioural Science Institute \\ Radboud University, Nijmegen \\ evelien.heijselaar@ru.nl
}

\author{
Tibor Bosse \\ Communication \& Media \\ Behavioural Science Institute \\ Radboud University, Nijmegen \\ t.bosse@ru.nl
}

\begin{abstract}
There is a new crisis emerging in human-agent interaction research: Instead of using validated questionnaires, individual studies are creating new questionnaires that claim to measure identical constructs. This makes replication studies and comparisons between studies near to impossible. In turn, meta-analyses to determine which characteristics are important to create agents that the user experiences as being intelligent are difficult to conduct. As part of the attempt to battle this crisis, in this current paper, we suggest the use of a Theory of Mind task to measure the implicit social behaviour users exhibit towards a virtual agent. In a two-part study, we present findings that suggest that participants conduct this Theory of Mind task as expected: participants adapt towards our virtual agent more than when they conduct the task alone. We additionally present preliminary results correlating performance in the Theory of Mind task to validated constructs. Unfortunately, our current results do not correlate significantly to the existing constructs. Data-collection is ongoing and hence no firm conclusions can be made about this second set of results. However, our data suggest that it is important to become aware that the existing validated constructs used in HCI research may not be tapping into what the researchers assume, and hence provide a basis for important discussions about these implications.
\end{abstract}

\section{KEYWORDS}

Human-computer interaction, Theory of Mind, Referential Communication Game

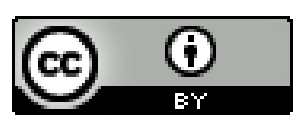

This work is licensed under a Creative Commons Attribution International 4.0 License.

IVA '21, September 14-17, 2021, Virtual Event, Japan

(C) 2021 Copyright is held by the owner/author(s).

ACM ISBN 978-1-4503-8619-7/21/09.

https://doi.org/10.1145/3472306.3478343
ACM Reference Format: Evelien Heyselaar and Tibor Bosse. 2021. Linking Theory of Mind to Validated Evaluations: Can Explicit Questionnaires Measure Implict Behaviour? In IVA '21: $A C M$ International Conference on Intelligent Virtual Agents Proceedings, September, 2021, Fukuchiyama, Japan. ACM, New York, NY, USA, 6 pages. https://doi.org/10.1145/3472306.3478343

\section{Introduction}

The overall goal of most intelligent virtual agent design is to create a character that the user interacts with as if it were another human. This would allow the agent to be seamlessly integrated into whatever its application: research, education, customer service, etc. If the agent is perceived as having humanlike cognitive abilities, the potential applications are endless. Even though research into which aspects are required to make an agent seem more human-like have answered many questions, currently it seems as if the final steps are always just out of reach.

One potential reason for this is the abundance of questionnaires that are being used. This has come about due to the continuous practice of creating new questionnaires instead of reusing validated questionnaires. In fact, a recent study conducted a review and identified 189 individual constructs (many measuring the same thing) being used [9]. $79 \%$ of these constructs were only used by a singly study. This means that it has become difficult to compare agents that are being used in different studies, more difficult to conduct replication studies, and near to impossible to conduct meta-analyses to determine which characteristics contribute significantly to creating a social agent that appears to have human-like cognitive abilities.

Additionally, the use of a questionnaire may not tap into what the researcher is attempting to measure. The term 'human-like' is quite vague. In most studies, when the term 'human-like' is used or a study measures the 'humanness' of an agent, it is synonymous to the assumption that the user attributes some sort of autonomous mind to the agent: that the agent is capable of feeling emotion, pain, independent thought, etc. This is also the definition we will use in this current paper when we use the term 'humanness': it is not about the appearance of the agent, but the perceived human-like cognitive ability of the agent. 
In 2018, a workgroup [8] was set-up in an attempt to merge these constructs together and determine how these constructs are linked together when interacting with an artificial social agent. This workgroup uses researcher-based methods: They ask the researchers to classify and re-classify constructs into different predefined groups, in an attempt to reduce the number of constructs and highlight underlying network(s). However, is such an explicit evaluation method the correct way to determine the 'key questionnaires' when evaluating an artificial social agent?

Everyday social interactions are mostly supported by implicit (automatic, unconscious) processes $[11,23]$. Hence it could be argued that an ecologically valid test of user's perception of 'humanness' should be based on implicit behaviour. This is echoed in the CASA theory $[26,29]$, the seminal theory that most social human-computer interaction is based on. The authors constantly empahsie that the underlying mechanism is mindlessness: participants are not away that they are responding socially to a computer. Therefore, is the use of explicit, researcher-based input the proper methodology to determine which questionnaires are relevant?

The goal of the current study is therefore two-fold: 1) Can we develop an implicit test to measure the perceived 'humanness' of an agent? and 2) Can we use the performance in this task to identify which validated constructs tap into this implicit measurement?

To meet the first aim, we propose to use a Theory of Mind task. Theory of Mind is the ability to attribute mental states (beliefs, intents, desires, emotions, knowledge, etc.) to others and to understand that others have beliefs, desires, and perspectives that are different from one's own [27]. This is the definition of what we want users to experience when they are interacting with an intelligent virtual agent. The task we propose has been conducted before in human-human experimental studies [31,32] and is also known as the 'referential communication game'. In this task, participants were seated in front of separate monitors with a divider so that they could not see each other. One participant was assigned to be the speaker, the other the listener. It was the task of the speaker to describe objects in an array in a way that the listener could identify it on their screen (Figure 1).

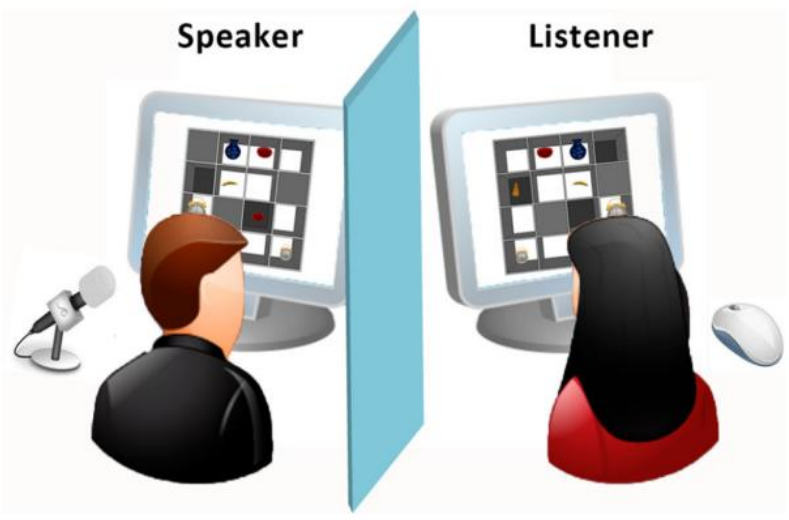

Figure 1: Overview of the human-human version of the Theory of Mind task. Speakers and listeners viewed opposite sides of arrays containing objects. Some objects were only visible to one of the participants. Adapted from [32].

The manipulation was such that on certain trials, the speaker was asked to describe one of three identical objects differing in size. One of these three objects was not visible to the listener, and the speaker was aware of this (Figure 2). Therefore, if the speaker considered the listener's perspective, they would describe the object in a way that ignores the third, "hidden" object, and therefore use the adjectives "big" or "small" when describing the object. However, if they did not consider the perspective of the listener, they would describe the object using the term "medium", as it is neither the "big" nor the "small" object. This would make it hard for the listener to identify which of the two objects they see is the "medium" one. There was no feedback, nor were clarification questions allowed, and thus the speaker could complete the task however they wanted, even if it made it difficult for the listener. Previous research has shown that taking the listener's perspective is more effortful, and therefore any adaptation is internally driven, self-motivated, and not automatic [32]. In the Vanlangendonck study [31], they showed that when participants complete this task together, the speaker adapts their responses so that the listener can better identify the objects $45.3 \%$ of the time. When the speaker conducted the same task alone, they would adapt their responses significantly less $(p<.001)$.

The Vanlangendonck study [31] also measured the brain activity of the speaker using fMRI while participants conducted this task, and showed significant activation in the medial prefrontal cortex (activated when thinking about the mental states of others) and in the temporoparietal junction (an area important for inferring the temporary states of other people, such as goals, intentions, and desires). The authors showed significant overlap in the brain activity when conducting the task and a Theory of Mind localizer. The study therefore illustrated that when conducting this task, participants "put themselves in their partner's shoes" when describing the objects. As this perspective taking is effortful, our 
assumption is that participants will only do this if they believe that their partner has a mental state to consider, i.e., a mind of their own.

In the current study, we will conduct a replication of the Vanlangendonck study, but have participants pair up with a virtual agent. If the participant believes that the virtual agent has a mental state that needs to be considered, then participants will adapt their description of the objects significantly more than when they conduct the task alone. We further predict that the proportion of adaptation will be less than those reported in Vanlangendonck and colleagues, as we do not assume that the virtual agent we use is humanlike enough to warrant that much adaptation.

For the second part of the current study, we will correlate the performance in the task to existing validated constructs. These constructs are taken from the 189 constructs identified in [9]. We therefore hope to be able to unify these constructs, to propose validated constructs that do tap into the perceived Theory of Mind of an intelligent virtual agent. Currently, data collection for this second experiment is ongoing, and 99 participants have thus far been collected. This allows us to provide some interesting preliminary findings for this second experiment.

\section{Method}

Although this study involves two separate experiments, the underlying methods are identical and hence the methodology for both will be reported here.

\subsection{Participants}

2.1.1 Experiment 1. Forty native Dutch speakers (29 female, $\mathrm{M}_{\mathrm{age}}$ : 21.98 years, SDage: 3.00) were recruited from the Radboud University Nijmegen student participant database. They gave digital informed consent before the start of the experiment. Participants were rewarded one credit or $€ 7,50$ for their participation. The study was approved by the Ethics Committee for the Faculty of Social Sciences of the Radboud University Nijmegen (ECSW-2018-117).

2.1.2 Experiment 2. Fifty-nine native Dutch speakers (47 female, Mage: 21.17 years, $S_{\text {age: }} 3.14$ ) were recruited from the university participant database.

2.1.3 Human data. The human data used in this analysis was collected in [31]. The participants were recruited between May and December 2013. Twenty native Dutch speakers (1 male, Mage: 22.55 years, range 19-28 years old) were recruited from the Radboud University participant database. The procedure mirrors the current procedure, except that the human participants did not fill in a questionnaire.

\subsection{Procedure}

Participants were invited to complete a task with a virtual agent. For Experiment 1, participants would complete the task with the virtual agent (VA; communicative condition) and alone (see below; non-communicative condition), the order of which was counterbalanced. For Experiment 2, the participants would complete the task only with the VA. For both experiments, after their interaction with the VA, they were instructed to fill in a questionnaire.

2.2.1 Referential Communication Game. The task is based on the one described in Vanlangendonck and colleagues [31,32].

Participants were informed that they would view an array of objects that they would have to describe for the VA. The VA, however, could only see the opposite side of said array (the "listener" in Figure 1). Each array contained 3 closed slots on either side.

Participants completed 120 trials. During the first phase of the trial (3000ms), the participant saw their side of the array. Then the participant was cued by means of a red circle around one of the objects, and described this object out loud. The cued object was always mutually visible. Participants were instructed not to use descriptions referring to the position of the object in the array, such as 'upper left corner' or 'rightmost'. Participants were not given any on-screen or verbal feedback about their performance, as is similar to the human version of this task. After their description, the participants heard a mouse click from the VA, indicating that they had selected an object from the other side of the array. No feedback was given and thus the participant did not know if the correct object had been identified by the VA. In actuality, the VA never selected an object, the mouse click was always played at the end of every trial.

2.2.2 Non-communicative referential communication game. This version was identical to the referential communication game, except participants completed this task without the VA present.

\subsection{Materials}

2.3.1 Referential Communication Game. The number, size, and visibility of the relevant objects were manipulated to create 6 conditions (Figure 2). For data analysis, only the Audience Design conditions are relevant, the other 4 conditions function as distractor trials. However, in order to include the human data collected from the original study, we included all conditions in our experiments to ensure the procedure was identical. For clarity, we will describe all conditions below:

2.3.1.1 Audience Design Condition. In the audience design conditions (left column, Figure 2), participants saw an extra competitor object that the VA could not see. In the obligatory audience design condition, participants saw 3 relevant identical objects of different sizes: One target object, one occluded competitor object, and one mutually visible object. The target object was always the medium-sized object of the 3 objects. If participants described this object from their own perspective, they would call it the medium object. On the other hand, if participants considered the perspective of the VA, they would ignore the occluded object and call the medium-sized object small or large. In the advisable audience design condition, participants saw 2 
relevant identical objects of different sizes: One target and one occluded competitor object. Given that the VA could see the target object but not the competitor object, participants did not have to use a contrasting size adjective.

2.3.1.2 Linguistic Control Condition. In the linguistic control condition (middle, Figure 2), the occluded object was replaced by another, unrelated object. As a result, participants saw one relevant object fewer in these conditions than in the audience design conditions. These were named linguistic control conditions, as participants were expected to produce the same description in these trials as on successful trials in the audience design conditions.

2.3.1.3 Visual Control Condition. In the visual control conditions (right, Figure 2), the object that was occluded in the audience design condition was visible to both the participant and the VA. As a result, both could see all relevant objects. These were named visual control conditions, because participants see the same number of relevant objects as in the audience design conditions. Neither of these control conditions re-quired the participant to take into account the perspective difference with the VA in order to communicate successfully. Trials from all 6 conditions were presented randomly, mirroring the Vanlangendonck study.

Twelve different empty virtual arrays were created. The arrays were filled with 6 to 8 objects chosen from a total of 22 objects selected from the Object Databank (courtesy of Michael Tarr Lab, Brown University, Providence, RI). Each object could appear in 4 different sizes to make sure that the participants could not rely on absolute size. Depending on the condition, participants saw 1, 2 or 3 relevant objects of the same type but different sizes. The remaining objects were fillers that also appeared in sets of 1, 2 or 3 objects of the same type to make sure participants could not predict which objects would be relevant. We made sure that the participants always saw the same total number of objects in a trial by adding additional filler objects to the occluded slots if needed.

For each participant, 120 trials were created with 20 repetitions across 6 conditions. Each participant was given the same object list, although it was randomized for each instance.

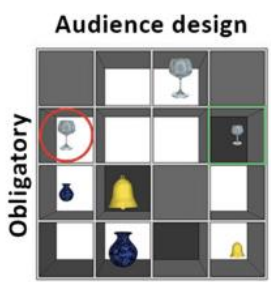

small/medium glass

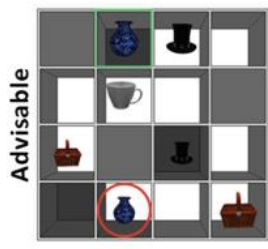

(small) vase

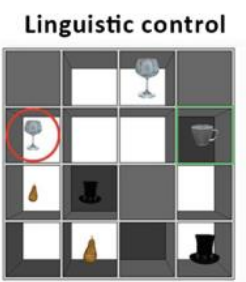

small glass

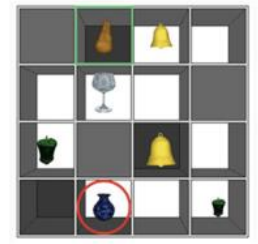

vase

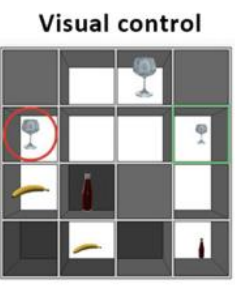

medium glass

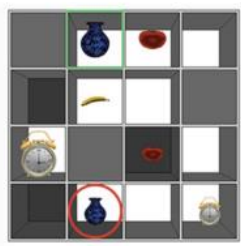

small vase
Figure 2: Overview of the six conditions from the participant's point of view, and the expected speaker responses. In the audience design conditions, speakers can either describe the target objects (circled in red) from their own perspective ("medium glass", "small vase"), or take into account the agent's perspective ("small glass", "vase"). There is no relevant perspective difference in the linguistic and visual control conditions. The green squares were added to the figure for clarification, and were not visible to the participants during the experiment. They indicate the objects that differ between conditions. Adapted from Vanlangendonck et al. [32].

2.3.2 Questionnaire. To explore any possible correlation between the participants performance in the referential communication game and an explicit evaluation, the participants were asked to complete a questionnaire after their completion of the task with the VA. The questionnaires used were taken from the collection of commonly used questionnaires to assess virtual agents $[9,10]$, specifically: Experience of Parasocial Interaction [14], Telepresence [24], Social Presence [1,21], Godspeed Scales [3], Satisfaction in Interaction, The Immersive Experience Questionnaire B [20], Interpersonal Similarity [33], Trust [13], Believability [6], Politeness [22], Engaging [30], Warmth [5], Working Alliance Inventory [19] and Dimensions of Mind Perception [DMP; 11]. The questionnaire consisted of 132 questions, which were presented randomly to the participant. Based on work by [7], we included the option "This question is too difficult to answer" to filter out questions that the participants did not find relevant to the current situation, as opposed to forcing them to answer.

2.3.3 Virtual Environment. The study was executed in the "Radboud Immersive Virtual Environment Research Lab" (RIVERlab) of the Behavioural Science Institute (BSI) at Radboud University. A head mounted display (HMD) was used to immerse the participant in the virtual environment. A sensor positioned on the top of the HMD recorded the participant's position and orientation in the room with 6 degrees of freedom at $120 \mathrm{~Hz}$. 
2.3.4. Virtual Agent. The agent's appearance suggested that she was a Causasian female in her mid-twenties, which matched the age and ethnicity of the Dutch speaker who recorded her speech.

Before the start of the communicative referential communication game, the virtual agent gave a brief introduction. The aim of this is to allow the participants to form an opinion of the VA. Previous studies have shown that this short introduction is enough to cause different opinions to influence implicit behaviour [17,18]. The same introduction length, content, and VA facial expressions were used as in [16,17]. Specifically, the agent blinked once every $1-5$ seconds (blink duration was 0.1 second). She smiled once every 5 -10 seconds and raised her eyebrows once every $1-5$ seconds. Therefore, even when the agent was not speaking, there were always subtle movements occurring, which helps the feeling of presence.

\subsection{Statistical Analysis}

2.4.1 Referential Communication Game. The output files were coded for adjective use. We created three variables: (1) Use of the adjective small/large, (2) use of no adjective, and (3) whether the descriptions reflected an adaptation to the VA's perspective. All variables were coded as the binomial variables 0 (no) and 1 (yes). For the results reported in this study, only the audience design conditions were used (left panel, Figure 2).

The data was analyzed using binomial mixed-effects models, using the lme4 package (version 1.1-23; [4] in $\mathrm{R}$ [28]). We used a maximal random-effects structure as was justified by the data [2]: The repeated-measured nature of the data was modelled by including a per-participant and per-item random adjustment to the fixed intercept ("random intercept"). We included all fixed effects as a random slope in the random intercept unless specified.

2.4.2 Factor Analysis The empties and 'This question is too difficult to answer' responses were replaced with the mean for that question using the forcats package (version 0.5.1; [34]).

Ideally, we would conduct an explorative factor analysis, however, due to the current low power of our study (99 participants to assess 132 questions), for this report we conducted a confirmatory factor analysis to recreate the original 17 variables. An a priori power analysis for a linear regression with 17 variables gives us a power of 0,8 for a sample size of 64 . The Shapiro-Wilks test revealed that some of the items are not normally distributed, for these variables we used a principal factor solution with an oblique rotation to correct for this. Only variables with a KMO test $>0.7$ were included. This resulted in only 7 variables remaining, which are listed in Table 1.
Table 1: Kaiser-Meyer-Olkin (KMO) Measure of Sampling Adequacy and Cronbach's Alpha for the 7 variables used in the analysis.

\begin{tabular}{lcc}
\multicolumn{1}{c}{ Variable } & KMO & Cronbach's alpha \\
\hline Telepresence & 0.72 & 0.74 \\
Self-Engagement & 0.70 & 0.67 \\
Engaging & 0.83 & 0.86 \\
Warmth & 0.76 & 0.75 \\
Bonding & 0.84 & 0.77 \\
DMP & 0.68 & \\
- Experience & & 0.86 \\
- Agency & & 0.69 \\
\hline
\end{tabular}

\section{$3 \quad$ Results}

\subsection{Experiment 1}

The aim of Experiment 1 was to determine whether the referential communication game would be suitable to measure Theory of Mind of a virtual agent. We additionally compared the data to that from the human version of the study [31].

The model included the interaction of the sum-contrasted fixed effects Condition (communicative (with VA) versus noncommunicative (solo); within subjects) and Agent (human versus VA; between subjects). Unfortunately, the human data did not code which objects the participants viewed, and hence only a perparticipant random intercept could be included. Table 2 reports the mixed model outcome.

Table 2: Summary of the binomial mixed effects model for the response Adaptation between the communicative and noncommunicative conditions, as well as human compared to virtual agent, for the referential communication game. Human data retrieved from [31].

\begin{tabular}{lrlrrl} 
& Coefficient & SE & $\boldsymbol{z}$ value & $\boldsymbol{p}$ value & \\
\hline Intercept & -0.87 & 0.35 & -2.53 & .011 & $*$ \\
Condition & 1.59 & 0.30 & 5.35 & $<.001$ & $* * *$ \\
Agent & 0.96 & 0.35 & 2.77 & .006 & $* *$ \\
Condition * Agent & 0.69 & 0.30 & 2.33 & .020 & $*$ \\
\hline
\end{tabular}

$\mathrm{N}=4797, \log$-likelihood $=-1501.9$

$* * *<.001 . * *<.01 . *<.05$

There is a significant difference between the proportion of adapted responses when the participants were conducting the task with a partner (communicative condition) compared to when they were conducting the task alone (non-communicative condition; $\beta=1.59$, $p<.001)$. There was also a significant difference between agents, such that participants adapted their responses more when they were conducting the task with a human (45.3\%), compared to participants who conducted the task with the VA $(29.9 \% ; \beta=.069$, $p=.020)$. 
Overall, this suggests that the referential communication game is transferrable to virtual partners.

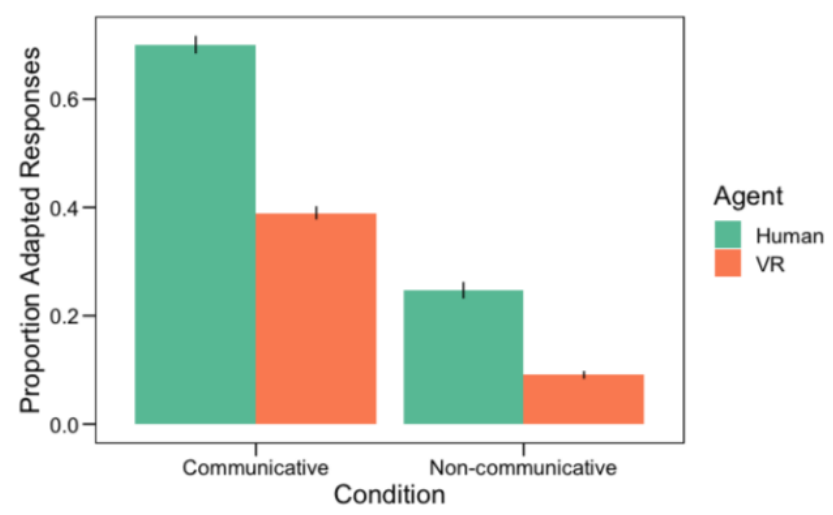

Figure 3. Proportion of adapted responses per Agent and Condition. Error bars represent standard error of the mean.

\subsection{Experiment 2}

The aim of Experiment 2 was to determine whether the proportion of adaptation in the referential communication game (i.e., the Theory of Mind the participant associates with their virtual partner) is correlated to any existing evaluative measures. This would therefore validate the existing measure as tapping into the perceived theory of mind of the virtual agent.

3.2.1 Experiment 1 vs Experiment 2. Before combining the two participant pools, we checked that the data was not significantly different from each other. We conducted a logit mixed effects model, with Experiment (1 versus 2) sum-contrasted. The data was not significantly different $(\beta=.40, p=.141)$. We are therefore able to combine our data to increase our dataset from 40 participants to 99.

3.2.2 Factor Analysis. A linear regression with all 7 variables (centered) and Adapt was conducted. None of the 7 variables correlated significantly with the amount the participant adapted their response for the virtual agent $(p>.127)$. Therefore, from the 7 existing variables, none are correlated to Theory of Mind, or specifically: to the performance in the Referential Communication Game.

\section{Conclusion}

The aim of the current study was two-fold: 1) Can Theory of Mind tasks be adapted for use with a virtual partner? and 2) Is performance in a theory of mind task correlated to existing measures?

Current research in the field of human-computer interaction, but especially intelligent virtual agents, is being overrun by different standardized questionnaires that all measure the same thing. This makes it very difficult to conduct meta-analyses to determine which characteristics are necessary to build a human-like social virtual agent. This study is one attempt to solve this problem. We defined the "humanness" of an agent as causing the participant to perceive the agent has having a human-like cognitive ability - "a mind of their own". We therefore adapted a social Theory of Mind paradigm, proven to work in human-human interaction studies $[31,32]$ for use with a digital virtual agent.

The first experiment in this study functioned as a 'proof of concept': Do participants adapt their responses to take the perspective of the virtual agent into account? Indeed, we found that this is case, with participants adapting their response by $30 \%$ when conducting the Theory of Mind task with a virtual agent compared to doing the task alone. This adaption was significantly less than the human-human results from [31], but this was expected. The virtual agent used in this study was very simple: the only 'humanlike' trait was randomly moving eyebrows and smiling. Although these facial expressions have been shown in previous work to produce the same implicit behavioural performance as with a human [16], the task was different, emphasizing the necessity of replicating a human-human task with a virtual agent.

We are confident that the Theory of Mind task used in the current study (the referential communication game) does tap into Theory of Mind networks, as shown with fMRI data [31]. However, recent neuroimaging work by Hortensius and colleagues (in press) have shown a disconnect between anthropomorpism in human-human tasks compared to human-computer tasks, as well as a disconnect between anthropomorphism and Theory of Mind. Therefore, even though the 'Computers are Social Actors' theory by Clifford Nass [25] shows that, behaviourally, we interact with computers as if they are humans, the research into the underlying brain networks are starting to contradict this. Further research using this Theory of Mind task should therefore include neuroimaging methods to determine whether the performance measured is created via the same Theory of Mind network as those in human-human interactions.

In the second experiment, an attempt was made to correlate the performance in the Theory of Mind task with existing measures. No correlations were found. Initially it appears that our measurements of these constructs were not as stable as expected: From the 17 constructs measured, only 7 had a KMO high enough to support factor analysis. This is possibly due to the method employed: all the questions were randomized across constructs, whereas usually these questions are presented within their respective construct clusters. This methodology was chosen as the analysis plan is an exploratory factor analysis, where we do not take existing constructs into account. However, data collection is on-going and with the current sample size, we do not have enough power to conduct the analysis this way.

Additionally, it could be that the 7 constructs do not correlate to our Theory of Mind measure, even with a full data-set. A key assumption is that the referential communication game is sensitive enough to show a difference in performance in minor changes in 
the intelligent virtual agent the participant is interacting with. In a previous study, we conducted this methodology with a chatbot [15], and although we showed the same significant differences, the proportion of adaption was less when participants interacted with the social chatbot (17.9\%) compared to when participants interacted with the virtual agent in the current experiment $(30.0 \%)$. It therefore seems that the referential communication game could be sensitive enough to measure these differences in the virtual partner, although future studies will continue to explore this assumption.

Overall, our study shows that, behaviourally, we can measure social Theory of Mind using a human-human developed task: The Referential Communication game. This 10-minute task is therefore a valuable way to implicitly measure how much mind a participant attributes to their human partner. With the ultimate goal of $\mathrm{HCI}$ research being to develop a virtual agent that participants interact with as if it were a human, this task will provide a valuable tool towards attaining that goal.

We had hoped to provide additional information as to which existing explicit measures may correlate with Theory of Mind. Unfortunately, from the 7 constructs analyzed (Telepresence, SelfEngagement, Engaging, Warmth, Bonding, and the Dimensions of Mind Perception) none appear to correlate to the performance in the Theory of Mind task. This is most likely due to the young nature of the study; there is still a lot of data to be collected before any concrete conclusions can be made.

Future studies will continue to build on the data-set, both within virtual agents, but also across agent types (chatbots and robots) in an attempt to determine which standardized measures could explain the difference in performance between these agents, with the overall aim of answering the question: which aspects cause an intelligent social agent to be perceived as having human-like cognitive abilities?

\section{REFERENCES}

[1] Theo Araujo. 2018. Living up to the chatbot hype: The influence of anthropomorphic design cues and communicative agency framing on conversational agent and company perceptions. Comput. Human Behav. 85, (2018), 183-189. DOI:https://doi.org/10.1016/j.chb.2018.03.051 Dale J Barr, Roger Levy, Christoph Scheepers, and Harry J Tily. 2013. Random effects structure for confirmatory hypothesis testing: Keep it maximal. J. Mem. Lang. 68, 3 (April 2013), 255-278. DOI:https://doi.org/10.1016/j.jml.2012.11.001

[3] Christoph Bartneck, Dana Kulić, Elizabeth Croft, and Susana Zoghbi. 2009. Measurement instruments for the anthropomorphism, animacy, likeability, perceived intelligence, and perceived safety of robots. Int. J. Soc. Robot. 1, 1 (2009), 71-81. DOI:https://doi.org/10.1007/s12369-0080001-3

[4] D. Bates, M Maechler, and Ben Bolker. 2012. lme4: Linear mixed-effects models using S4 classes (2011). R package version 0.999375-42. (2012).

[5] Kirsten Bergmann, Friederike Eyssel, and Stefan Kopp. 2012. A second chance to make a first impression? how appearance and nonverbal behavior affect perceived warmth and competence of virtual agents over time. Lect. Notes Comput. Sci. (including Subser. Lect. Notes Artif. Intell. Lect. Notes Bioinformatics) $7502 \quad$ LNAI, (2012), 126-138. DOI:https://doi.org/10.1007/978-3-642-33197-8_13

[6] Elisabetta Bevacqua, Igor Stanković, Ayoub Maatallaoui, Alexis Nédélec, and Pierre De Loor. 2014. Effects of coupling in human-virtual agent body interaction. Lect. Notes Comput. Sci. (including Subser. Lect. Notes Artif.
Intell. Lect. Notes Bioinformatics) 8637 LNAI, (2014), 54-63. DOI:https://doi.org/10.1007/978-3-319-09767-1_7

[7] Meia Chita-Tegmark, Theresa Law, Nicholas Rabb, and Matthias Scheutz. 2021. Can you trust your trust measure? ACM/IEEE Int. Conf. HumanRobot Interact. (2021), 92-100. DOI:https://doi.org/10.1145/3434073.3444677

[8] Siska Fitrianie, Merijn Bruijnes, Willem-paul Brinkman, Amal Abdulrahman, Deborah Richards, Andrea Bönsch, ..., and M Tsfasman. 2018. Artificial Social Agent Evaluation Instrument. Retrieved from Retrieved from osfio/6duf7

[9] Siska Fitrianie, Merijn Bruijnes, Deborah Richards, Amal Abdulrahman, and Willem Paul Brinkman. 2019. What are we measuring anyway? A literature survey of questionnaires used in studies reported in the intelligen virtual agent conferences. In ACM International Conference on Intelligent Virtual Agents (IVA '19), July 2-5, ACM, New York, NY, USA, Paris, France.

[10] Siska Fitrianie, Merijn Bruijnes, Deborah Richards, Andrea Bönsch, and Willem Paul Brinkman. 2020. The 19 Unifying Questionnaire Constructs of Artificial Social Agents: An IVA Community Analysis. In Proceedings of the 20th ACM International Conference on Intelligent Virtual Agents (IVA '20), October 19-23, ACM, New York, NY, USA. DOI:https://doi.org/10.1145/3383652.3423873

[11] Chris D Frith and Uta Frith. Mechanisms of Social Cognition. DOI:https://doi.org/10.1146/annurev-psych-120710-100449

[12] Heather M Gray, Kurt Gray, and Daniel M Wegner. 2007. Dimensions of mind perception. Science 315, 5812 (February 2007), 619 DOI:https://doi.org/10.1126/science.1134475

[13] Rosanna E. Guadagno, Jim Blascovich, Jeremy N. Bailenson, and Cade Mccall. 2007. Virtual humans and persuasion: The effects of agency and behavioral realism. Media Psychol. 10, 1 (2007), 1-22. DOI:https://doi.org/10.108/15213260701300865

[14] Tilo Hartmann and Charlotte Goldhoorn. 2011. Horton and Wohl revisited: Exploring viewers' experience of parasocial interaction. J. Commun. 61, 6 (2011), 1104-1121. DOI:https://doi.org/10.1111/j.14602466.2011.01595.x

[15] Evelien Heyselaar and Tibor Bosse. 2020. Using Theory of Mind to Assess Users' Sense of Agency in Social Chatbots. Lect. Notes Comput. Sci. (including Subser. Lect. Notes Artif. Intell. Lect. Notes Bioinformatics) 11970 LNCS, (2020), 158-169. DOI:https://doi.org/10.1007/978-3-03039540-7 11

[16] Evelien Heyselaar, Peter Hagoort, and Katrien Segaert. 2015. In dialogue with an avatar, language production is identical compared to dialogue with a human partner. Behav. Res. Methods 49, 1 (December 2015), 1-15 DOI:https://doi.org/10.3758/s13428-015-0688-7

[17] Evelien Heyselaar, Peter Hagoort, and Katrien Segaert. 2017. How social opinion influences syntactic processing-An investigation using virtual reality. PLoS One 12, 4 (April 2017), e0174405. DOI:https://doi.org/10.1371/journal.pone.0174405

[18] Evelien Heyselaar, Ali Mazaheri, Peter Hagoort, and Katrien Segaert. 2018. Changes in alpha activity reveal that social opinion modulates attention allocation during face processing. Neuroimage 174, (July 2018), 432-440. DOI:https://doi.org/10.1016/j.neuroimage.2018.03.034

[19] Adam O. Horvath and Leslie S. Greenberg. 1989. Development and validation of the Working Alliance Inventory. J. Couns. Psychol. 36, 2 (1989), 223-233. DOI:https://doi.org/10.1037//0022-0167.36.2.223

[20] Charlene Jennett, Anna L. Cox, Paul Cairns, Samira Dhoparee, Andrew Epps, Tim Tijs, and Alison Walton. 2008. Measuring and defining the experience of immersion in games. Int. J. Hum. Comput. Stud. 66, 9 (2008), 641-661. DOI:https://doi.org/10.1016/j.ijhcs.2008.04.004

[21] Kwan Min Lee, Wei Peng, Seung A. Jin, and Chang Yan. 2006. Can robots manifest personality?: An empirical test of personality recognition, social responses, and social presence in human-robot interaction. J. Commun. 56, 4 (2006), 754-772. DOI:https://doi.org/10.1111/j.14602466.2006.00318.x

[22] Mark Ter Maat, Khiet P. Truong, and Dirk Heylen. 2010. How turn-taking strategies influence users' impressions of an agent. Lect. Notes Comput. Sci. (including Subser. Lect. Notes Artif. Intell. Lect. Notes Bioinformatics) $6356 \quad$ LNAI, March $2017 \quad$ (2010), 441-453. DOI:https://doi.org/10.1007/978-3-642-15892-6_48

[23] Tanaz Molapour, Cindy C Hagan, Brian Silston, Haiyan Wu, Maxwell Ramstead, Karl Friston, Dean Mobbs, California Blvd, Brain Program, and California Blvd. Seven Computations of the Social Brain. 1-46.

[24] Jesper Mortensen, Vinoba Vinayagamoorthy, Mel Slater, Anthony Steed, Mary C Whitton, and Benjamin Lok. 2002. Collaboration in TeleImmersive Environments. Proc. Eight Eurographics Work. Virtual Environ. (2002), 93-101.

[25] Clifford Nass and Youngme Moon. 2000. Machines and Mindlessness: Social Responses to Computers. J. Soc. Issues 56, 1 (January 2000), 81- 
103. DOI:https://doi.org/10.1111/0022-4537.00153

[26] Clifford Nass, Jonathan Steuer, and Ellen R Tauber. 1994. Computers are Social Actors. (1994), 72-78.

[27] David Premack and Guy Woodruff. 1978. Does the chimpanzee have a theory of mind? Behav. Brain Sci. 4, (1978), 515-526.

[28] R Core Development Team. 2011. R: A language and environment for statistical computing. Vienna, Austria.

[29] Byron Reeves and Clifford Nass. 1997. The Media Equation: How people treat computers, television, and new media like real people and places. Association for Computing Machinery, New York, NY.

[30] Lazlo Ring, Dina Utami, Stefan Olafsson, and Timothy Bickmore. 2016. Increasing engagement with virtual agents using automatic camera motion. Lect. Notes Comput. Sci. (including Subser. Lect. Notes Artif. Intell. Lect. Notes Bioinformatics) 10011 LNAI, (2016), 29-39. DOI:https://doi.org/10.1007/978-3-319-47665-0_3

[31] Flora Vanlangendonck, Roel M Willems, and Peter Hagoort. 2018. Taking common ground into account: Specifying the role of the mentalizing network in communicative language production. PLoS One 13, 10 (October 2018),

e0202943.

DOI:https://doi.org/10.1371/journal pone.0202943

[32] Flora Vanlangendonck, Roel M Willems, Laura Menenti, Peter Hagoort, Flora Vanlangendonck, Roel M Willems, Laura Menenti, and Peter Hagoort. 2016. An early influence of common ground during speech planning. 3798 ,

(2016). DOI:https://doi.org/10.1080/23273798.2016.1148747

[33] Kodi Weatherholtz, Kathryn Campbell-Kibler, and T Florian Jaeger. 2014. Socially-mediated syntactic alignment. Lang. Var. Change 26, 3 (October 2014), 387-420. DOI:https://doi.org/10.1017/S0954394514000155

[34] Hadley Wickham. 2021. Package ' forcats .' 